Editorial

\title{
Acknowledgement to Reviewers of Land in 2019
}

\section{Land Editorial Office}

MDPI, St. Alban-Anlage 66, 4052 Basel, Switzerland; land@mdpi.com

Published: 16 January 2020

The editorial team greatly appreciates the reviewers who have dedicated their considerable time and expertise to the journal's rigorous editorial process over the past 12 months, regardless of whether the papers are finally published or not. In 2019, a total of 191 papers were published in the journal, with a median time to first decision of 13 days and a median time from submission to publication of 42 days. The editors would like to express their sincere gratitude to the following reviewers for their generous contribution in 2019:

Abdel-Hamid, Ayman

Abdullah, Abu Yousuf Md

Abrams, Jesse

Abubakar, Ismaila Rimi

Abuzar, Mohammad

Aceituno-Mata, Laura

Acharya, Ram Prasad

Adhikari, Sanchayeeta

Adhikari, Shankar

Aguilar-Stoen, Mariel

Ahmed, Khaled Galal

Akhtar-Schuster, Mariam

Akoto, Daniel S.

Albaladejo, Juan

Albert, Christian

Aledo, Antonio

Alfasi, Nurit

Alfonso Piña, William H.

Ali, Abid

Allam, Zaheer

Allen, Karen

Alo, Clement A

Alonso, Gema Cárdenas

Amato, Federico

Anchang, Julius Y.

Andrzej, Mazur

Ango, Tola Gemechu

Antichi, Daniele
Lujan-Alvarez, Concepcion

Luo, Jun

Luo, Pingping

Lv, Zhi-Yong

Lyons, Seán

Madau, Fabio A.

Mahdianpari, Masoud

Mahmoud, Mahmoud Ibrahim

Majewski, Edward

Malak-Rawlikowska, Agata

Malek, Žiga

Man Li, Rita Yi

Manzano, Pablo

Mao, Liang

Marks-Bielska, Renata

Marques, Bruno

Marques, Maria Jose

Martellozzo, Federico

Martinez Paz, Jose Miguel

Martínez-López, Javier

Mas Caussel, Jean-François

Mashimbye, Zama Eric

Masot, Ana Nieto

Mastelić Ivić, Siniša

Masuhara, Naoki

Matthews, Alan

Mátyás, Csaba

Maurizia, Dr. Sigura 


\begin{tabular}{|c|c|}
\hline Antinoro, Chiara & Mauro, Giovanni \\
\hline Asiama, Kwabena & Mazzeo, Giuseppe \\
\hline Asiama, Kwabena & McCord, Michael \\
\hline Aspinall, Richard & McEldowney, John \\
\hline Athanasiadou, Eleni & Mdee, Anna \\
\hline Autrey, Bradley & Mehan, Sushant \\
\hline Azadi, Hossein & Melendez-Pastor, Ignacio \\
\hline Babb, Courtney & Mendoza-Fernández, Antonio \\
\hline Baffoe, Gideon & Mercuri, Anna María \\
\hline Baird, Ian & Merola, Simona Silvia \\
\hline Balawejder, Monika & Mertz, Ole \\
\hline Baležentis, Tomas & Michalik-Śnieżek, Malwina \\
\hline Barasa, Bernard & Michaud, Jean \\
\hline Barbarossa, Luca & Midmore, David \\
\hline Barnett, John & Mietkiewicz, Nathan \\
\hline Barry, Michael & Migliorini, Sara \\
\hline Bavorová, Miroslava & Mikita, Tomáš \\
\hline Bayrak, Mucahid & Millington, Andrew \\
\hline Bayrak, Mucahid & Miyazaki, Hiroyuki \\
\hline Beaudry, Frederic & Moccia, Francesco Domenico \\
\hline Becerril, Hilario & Molho, Jeremie \\
\hline Beckmann, Volker & Molnar, Gyozo \\
\hline Bedoya-Perales, Noelia S. & Moreno-Sanchez, Rafael \\
\hline Beeri, Ofer & Móricz, Norbert \\
\hline Bełej, Mirosław & Morrone, Domenico \\
\hline Bell, Simon & Mouysset, Lauriane \\
\hline Berezuk, André Geraldo & Muchová, Zlatica \\
\hline Berhane, Tedros M. & Mukul, Sharif Ahmed \\
\hline Betge, David & Müller, Bernhard \\
\hline Bhowmik, Arnab & Munoz-Rojas, Jose \\
\hline Bickford, Nate & Munteanu, Catalina \\
\hline Biddoccu, Marcella & Muresan, Iulia Cristina \\
\hline Bieda, Agnieszka & Murgante, Beniamino \\
\hline Biel, Robert & Murillo-Sandoval, Paulo \\
\hline Bielska, Anna & Murzin, Anton \\
\hline BILAL, Muhammas & Myga-Piątek, Urszula \\
\hline Biłozor, Andrzej & Nagaike, Takuo \\
\hline Bird, Neil & Napoli, Grazia \\
\hline Blecharczyk, Andrzej & Nchanji, Eileen Bogweh \\
\hline Bogunovic, Igor & Ndoro, Jorine \\
\hline Bojnec, Stefan & Newlands, Nathaniel K. \\
\hline Bolland, Luciana Porter & Newman, Lenore \\
\hline Bollig, Michael & Ngutu, Mariah \\
\hline
\end{tabular}




\begin{tabular}{|c|c|}
\hline Bonifazi, Alessandro & Nguyen, Lan Hoang \\
\hline Bonifazi, Alessandro & Nguyen, Trung H. \\
\hline Bontoux, Laurent & Nicu, Ionut Cristi \\
\hline Bourassa, Steven C. & Niedbała, Gniewko \\
\hline Briassoulis, Helen & Nielsen, Jonas \\
\hline Brown, Calum & Nita, Andreea \\
\hline Brown, William D. & Nocca, Francesca \\
\hline Brunori, Gianluca & Nolè, Gabriele \\
\hline Bryant, Christopher & Nones, Michael \\
\hline Buchmann, Carsten & Norton, George \\
\hline Bulteau, Julie & Nunes, Adélia \\
\hline Burian, Jaroslav & Nunes, Leonel \\
\hline Burrell, Arden & Ocampo Talero, Angelica Maria \\
\hline Bydłosz, Jarosław & Ochoa-Moreno, Wilman-Santiago \\
\hline Cabral-Pinto, Marina M. S. & Ogawa, Kazuharu \\
\hline Caiserman, Arnaud & Ogneva-Himmelberger, Yelena \\
\hline Calaza, Pedro & Ogwang, Tom \\
\hline Caldarice, Ombretta & Olanya, David \\
\hline Calvelo-Pereira, Roberto & Olfat, Hamed \\
\hline Cano-Orellana, Antonio & Oliveira, Gustavo \\
\hline Cantisani, Giuseppe & Ong, Boon \\
\hline Cao, Jianjun & Ongolo, Symphorien \\
\hline Caron, Cynthia & Orzolek, Michael D. \\
\hline Carpentieri, Gerardo & Oswald, John \\
\hline Carvalho, Ana Maria Pinto & Pablo-Romero, María \\
\hline Casse, Thorkil & Paci, Chris \\
\hline Castanho, Rui Alexandre & Pagani, Marco \\
\hline Castellini, Mirko & Palang, Hannes \\
\hline Castro, A. Peter & Palazzo, Anna Laura \\
\hline Cavallo, Eugenio & Palme, Massimo \\
\hline Cecchin, Andrea & Palumbo, Elisabetta \\
\hline Cerdà, Artemi & Pamučar, Dragan \\
\hline Cerdà, Artemi & Pan, Haozhi \\
\hline Cerdà, Artemi & Panagopoulos, Thomas \\
\hline Cerdà, Artemio & Pandey, Gunjan \\
\hline Cevasco, Roberta & Panek, Jiri \\
\hline Chatzitheodoridis, Fotios & Paneque, Manuel \\
\hline Chen, Bin & Panford, Kwamina \\
\hline Chen, Ji & Papadopoulos, Apostolos G. \\
\hline Chen, Liang & Parcero-Oubiña, César \\
\hline Chen, Yong & Pascher, Kathrin \\
\hline Chenal, Jérôme & Patel, Nirav Nikunj \\
\hline Chew, Robert F. & Patrick Connors, John \\
\hline
\end{tabular}


Chigbu, Eugene

Chigbu, Uchendu Eugene

Chowdhury, AFM Kamal

Christman, Zachary

Ciglič, Rok

Cipollina, Maria

Cirella, Giuseppe T.

Clark, Sean

Colson, Gregory

Comanescu, Laura

Conley, Jamison

Conner, David

Conner, David

Connors, John Patrick

Corbelle Rico, Eduardo Jose

Coscieme, Luca

Coughlan, Michael

Coughlan, Michael R.

Cramb, Rob

Cuffaro, Nadia

Cunliffe, Emma

Cunliffe, Emma

Curatola Fernández, Giulia Floriana

Čvoro, Malina

Da Silva, Isabel Martinho

Dąbrowska, Jolanta

Datta, Rahul

Dawes, Warrick R.

Day, Terence

De Jong, Wil

De Maria, Marcello

De Melo Cartaxo, Tiago

De Toro, Pasquale

De Toro, Pasquale

De Valck, Jeremy

De Vries, Walter T.

Del Barrio, Gabriel

Del Bo, Chiara

Delotto, Roberto

Delshammar, Tim

Demartini, Eugenio

Deng, Jifeng

Detsis, Vassilis
Pătru-Stupariu, Ileana

Pavlickova, Katarina

Payne, Geoffrey

Paynter, Ian

Pearson, Diane

Peguero-Pina, José Javier

Pekney, Natalie

Pellegrini, Paola

Peng, Jingjing

Pérez González, María Eugenia

Perito, Maria Angela

Petrisor, Alexandru-Ionut

Petropoulou, Chryssanthi (Christy)

Petrovič, František

Pham, Binh Thai

Picos, Juan

Pietkiewicz, Michał

Pinedo-Alvarez, Alfredo

Pivić, Radmila

Pizzi, Simone

Pokhrel, Bijay

PONCE SOLE, JULIO

Ponomarev, Eugene

Potts, Ruth

Pradhan, Prajal

Precup, Radu-Emil

Prescott, Graham

Prestele, Reinhard

Pribadi, Didit Okta

Price, Bronwyn

Prishchepov, Alexander

Privitera, Donatella

Prosperi, Paolo

Pulido Fernández, Manuel

Pulighe, Giuseppe

Punalekar, Suvarna

Qi, Yi

Quinn, John E.

Raap, Edwin

Radović Marković, Mirjana

Radu-Daniel, Pintilii

Rahman, Muhammad Tauhidur

Rak, Janusz 


\begin{tabular}{|c|c|}
\hline Dewan, Ashraf & Ramírez, M. Isabel \\
\hline Dewan, Ashraf M. & Ramkissoon, Haywantee \\
\hline Dhital, Narayan Prasad & Ranagalage, Manjula \\
\hline Di Bene, Claudia & Raouf, Abdul \\
\hline Diaz-Sarachaga, Jose Manuel & Rappe, Erja \\
\hline Dietrich, Jörg & Raši, Rastislav \\
\hline Dinssa, Etefa Guyassa & Rausch, Lisa \\
\hline Dissanayake, DMSLB & Recanatesi, Fabio \\
\hline Dissanayake, DMSLB & Reed, Kevin F.M. \\
\hline Dobbs, Cynnamon & Reis, Mariane S. \\
\hline Dóniz-Páez, Javier & Reisi, Marzieh \\
\hline Donkor, Emmanuel & Rescia, Alejandro \\
\hline Duan, Hanchen & Ribeiro, Rita Cláudia Cardoso \\
\hline Edrisi, Sheikh & Ricart Casadevall, Sandra \\
\hline Ekblom, Anneli & Ricart, Sandra \\
\hline Elbl, Jakub & Riccioli, Francesco \\
\hline Elia, Elikkos & Richter, Kai-Florian \\
\hline Elsasser, Peter & Ridder, Elizabeth \\
\hline Erokhin, Vasilii & Riekkinen, Kirsikka \\
\hline Escudero-Gómez, Luis Alfonso & Riikonen, Anu \\
\hline Evans, Tracy R. & Rijal, Santosh \\
\hline Favas, Paulo & Ristić Trajković, Jelena \\
\hline Faye, El Hadji & Robinson, Derek T. \\
\hline Fedorov, Alexander & Rodella, Ilaria \\
\hline Feltynowski, Marcin & Rodrigo-Comino, Jesus \\
\hline Feng, Quanlong & Rodrigues, Evelina \\
\hline Ferreira, Hugo Enrique Júnez & Roić, Miodrag \\
\hline Ficko, Andrej & Roitman, Sonia \\
\hline Figueiredo, Carlos & Rolando, Diana \\
\hline Filchev, Lachezar & Román-Cascón, Carlos \\
\hline Filion, Pierre & Rosa, Isabel \\
\hline Fiorini, Lorena & Rosca, Sanda \\
\hline Fisher, Jolene & Rosenberg, Elissa \\
\hline Flachsbarth, Insa & Rosina, Konstantin \\
\hline Florenzano, Assunta & Ross, Andrea \\
\hline Foggin, Marc & Rotondo, Francesco \\
\hline Folgado-Fernández, José Antonio & Ruda, Aleš \\
\hline Folgôa Batista, Maria Teresa & Ruiz, Cristián A. Ducoing \\
\hline Fonte, Maria & Russo, Alessio \\
\hline Forsyth, Tim & Rusu, Teodor \\
\hline Fox, Jefferson & Rusu, Teodor \\
\hline Friedrich, Theodor & Rutten, Rosanne \\
\hline Frueh-Mueller, Andrea & Rydell, Jens \\
\hline
\end{tabular}




\begin{tabular}{|c|c|}
\hline Fujji, Yasuyuki & Rzasa, Krzysztof \\
\hline Gallemore, Caleb & Sabina, Źróbek \\
\hline Gao, Jinlong & Safonte, Fabiola \\
\hline Garau, Chiara & Sałabun, Wojciech \\
\hline Garay Tamajón, Lluís & Salata, Stefano \\
\hline Gascón, José Luis Gurría & Salmerón-Manzano, Esther \\
\hline Gasparatos, Dionisios & Salvati, Luca \\
\hline Gasparatos, Dionisios & Salvati, Luca \\
\hline Gava, Oriana & Salvia, Rosanna \\
\hline Gavrilidis, Athanasios-Alexandru & Samaha, Salma \\
\hline Gedefaw, Abebaw Andarge & San Martin, Narkis Morales \\
\hline Gellert, Paul K. & Sandham, Luke \\
\hline German, Laura & Sano, Edson Eyji \\
\hline Geronimo, Rollan C. & Santana Cordero, Aarón M. \\
\hline Ghawana, Tarun & Šantić, Danica \\
\hline Ghinoi, Stefano & Santoro, Antonio \\
\hline Gkiougkis, Ioannis & Sanyé-Mengual, Esther \\
\hline GLAVAN, Matjaž & Sareen, Siddharth \\
\hline Gojda, Martin & Sarría, Francisco Alonso \\
\hline Golub, Alexander & Savin, Igor Yu. \\
\hline Gomes, Alessandra & Schelhas, John \\
\hline Gomes, Eduardo & Schmidt-Vogt, Dietrich \\
\hline Goodin, Douglas G. & Schmidt-Vogt, Dietrich \\
\hline Gorbenkova, Elena & Schneibel, Anne \\
\hline Górka, Anna & Schneider, Laura \\
\hline Graham, Norman & Scorzini, Anna Rita \\
\hline Gray, Doris & Scullion, Jason \\
\hline Green, Andrew & Secondi, Luca \\
\hline Gregg, Jay & ŞEKERTEKİN, Aliihsan \\
\hline Grima, Nelson & Sena, Kenton \\
\hline Guevara, Mario & Senwo, Zachary \\
\hline Gullino, Paola & Serbin, Guy \\
\hline Guo, Lijia & Seymoure, Brett M \\
\hline Gyawali, Buddhi & Sharma, Harmandeep \\
\hline Haack, Barry & Shirvani Dastgerdi, Ahmadreza \\
\hline Haginoya, Shigenori & Shrestha, Him L. \\
\hline Hahn, Allison & Silva-Gómez, Sonia Emilia \\
\hline Hailemariam, Sisay & Simo, Alex Van Der Meer \\
\hline Hammad, Ahmed & Simoniello, Tiziana \\
\hline Hamman, Sarah T. & Skokanová, Hana \\
\hline Hanus, Paweł & Skotnicka-Zasadzień, Bożena \\
\hline $\mathrm{Hao}, \mathrm{Pu}$ & Soler, Luciana S. \\
\hline Haoyuan, Hong & Sompolska-Rzechuła, Agnieszka \\
\hline
\end{tabular}


Haruna, Samuel

Harwood, Christopher

Hasanagas, Nikolaos D.

Hassan, Mohammad Mehedy

Haughton, Jonathan

Hazbavi, Zeinab

Hebinck, Paul

Heckman, Joseph

Heikinheimo, Vuokko

Hein, Nils

Hentze, Konrad

Herrero, Baudilio

Hett, Cornelia

Higginbottom, Thomas P.

Hinten, Melissa

Hoffmann, Ellen M.

Högbom, Lars

Holloway, Paul

Home, Robert

Hoops, Björn

Horst, Megan

Houghton, Richard A

Hseu, Zeng-Yei

$\mathrm{Hu}$, Richard

Huang, Haiyan

Huizenga, Daniel

Huq, Nazmul

Huston, Simon

Hvenegaard, Glen

Hynes, Mike

Im, Sangjun

Imbrenda, Vito

Insch, Andrea

Ioan Cristian, Ioja

Ioannou, Konstantinos

Ishikawa, Daitaro

Jaba, Elisabeta

Jakab, Gergely

Jandl, Robert

Janus, Jaroslaw

Jauker, Frank

Javeed, Nadeem

Jitea, Ionel Mugurel
Soundy, Andrew

Špulerová, Jana

Sroka, Wojciech

Ståhl, Göran

Stathis, Dimitrios

Stavi, Ilan

Steenhuis, Tammo

Stöber, Silke

Stolte, Jannes

Stolze, Susann

Stręk, Żanna

Strimbu, Bogdan M

Stubkjær, Erik

Sturgeon, Janet

Sturman, Kathryn

Štych, Přemek

SU, Shiliang

Sun, Weiwei

Sung, Jongsang

Suzack, Cheryl

Svartengren, Magnus

Swagemakers, Paul

Swiader, Malgorzata

Sypion-Dutkowska, Natalia

Szilassi, Peter

Szulczewska, Barbara

Takac, Ivan

Tara, Ata

Tarantino, Cristina

Teodoro, Paulo Eduardo

Thorn, Alexandra M.

Tolleson, Doug

Tomal, Mateusz

Tonello, Kelly

Torre, Carmelo Maria

Torre, Carmelo Maria

Torres, Gerardo

Toscano, Pietro

Toulmin, Camilla

Tracey, Skillington

Travlos, Ilias

Trojanek, Radoslaw

Trovato, Maria Rosa 


\begin{tabular}{|c|c|}
\hline Jobbágy, Ján & Tsikudo, Kwame A. Adovor \\
\hline Joksimović, Marko & Unangst, Jennifer \\
\hline Joslin, Audrey & Unda, Mariana \\
\hline Jug, Irena & Unks, Ryan \\
\hline Julius, Mangisoni & Usai, Alessia \\
\hline Kaim, Dominik & Uwayezu, Ernest \\
\hline Kaletova, Tatiana & Valentin, Herbei Mihai \\
\hline Kapović-Solomun, Marijana & Valette, Elodie \\
\hline Kariuki, Rebecca & Vallini, Giovanni \\
\hline Katunský, Dušan & Van der Haar, Gemma \\
\hline Kefalas, George & Van der Werf, Wopke \\
\hline Kholodov, Vladimir & Van Gasselt, Stephan \\
\hline Kim, Hyun Woo & Van Leeuwen, Boudewijn \\
\hline Kim, Hyungkyoo & Van Oosterzee, Penny \\
\hline Kim, Minseo & Van Wensem, Joke \\
\hline Kim, Soonki & Vanclay, Frank \\
\hline Kindu, Mengistie & Vanni, Francesco \\
\hline King, David A. & Vant-Hull, Brian \\
\hline Kinoshita, Tsuguki & Verhagen, Philip \\
\hline Kitissou, Marcel & Verschoor, Gerard \\
\hline Kizos, Thanasis & Viana, Cláudia M. \\
\hline Kleemann, Janina & Vicente-Vicente, José Luis \\
\hline Klein, Johannes & Vicente-Vicente, José Luis \\
\hline Klimach, Anna & VÎRGHILEANU, Marina-Ramona \\
\hline Koech, Richard & Vlahos, Georgios \\
\hline Koglo, Yawovi S. & Vogt, Kristiina \\
\hline Kolecka, Natalia & Von Maltitz, Graham \\
\hline Köppel, Johann & Votsi, Nefta-eleftheria P. \\
\hline Kosmas, Kostas & Vukomanovic, Jelena \\
\hline Kostov, Philip & Vuletić, Dijana \\
\hline Krajewski, Piotr & Vyzoviti, Sophia \\
\hline Krakauer, Nir Y. & Walcek, Chris \\
\hline Krigsholm, Pauliina & Walker, Victoria \\
\hline Krikser, Thomas & Walters, Bradley \\
\hline Kronenburg García, Angela & Walters, Peter \\
\hline Kubalíková, Lucie & Walton, Andrea \\
\hline Kuffer, Monika & Wandsnider, Luann \\
\hline Kuntashula, Elias & Wang, Hao \\
\hline Kuntz, Steffen & Wang, Panshi \\
\hline Kuo, Hsing-Fu & Wang, Shaohua \\
\hline Kupidura, Przemysław & Warshawsky, Daniel N. \\
\hline Kurppa, Sirpa & Waters, Tony \\
\hline Lagerqvist, Yayoi & West, Paul \\
\hline
\end{tabular}


Lambini, Cosmas Kombat

Larcher, Federica

Larjavaara, Markku

Latkowska, Monika

Lebailly, Philippe

Lee, Yuh-Ming

Lehavi, Amnon

Leisz, Stephen J.

Lemoine, Guido

León Araya, Andrés

Leone, Antonio

Leone, Federica

Li, Shicheng

Li, Yingru

Li, Zhongjin

Lin, Hebin

Liu, Tao

Liu, Tie

Liu, Wan-Yu

Liuzzo, Lorena

Lojka, Bohdan

Longato, Davide

Lorilla, Roxanne Suzette

Louman, Bas

Loures, Luis

Loures, Luis

Lozano-Baez, Sergio E.

Lu, Dau-Jye

Luc, Malgorzata

Ludwig, Grit
Whiteley, Hugh

Wiesmeier, Martin

Wily, Liz Alden

Winfrey, Brandon K.

$\mathrm{Wu}$, Changshan

Wulder, Michael

$\mathrm{Xu}$, Yaping

Yamamoto, Kristina

Yee, Susan

Yezer, Anthony

Yilmaz, Kuzey

Yong, Jean W. H.

Yu, Yanyan

Zakrzewska-Półtorak, Alicja

Zambon, Ilaria

Zemke, Julian

Zevenbergen, Jaap

Zgłobicki, Wojciech

Zhang, Qi

Zhang, Weixing

Zhang, Xingnan

Zhao, Wei

Zheng, Yuanrun

Zhong, Taiyang

Zoppi, Corrado

Źróbek, Ryszard

Źróbek-Różańska, Alina

Żukowski, Arkadiusz

Zullo, Francesco

Zurqani, Hamdi A 